\title{
A practical approach for identifications based on mycotoxin characters of Penicillium
}

\author{
R. Russell M. Paterson ${ }^{1,2}$, Armando Venâncio ${ }^{1}$ and Nelson Lima ${ }^{1,2}$
}

${ }^{\prime}$ Centro de Engenharia Biológica and ${ }^{2}$ Micoteca da Universidade do Minho, Universidade do Minho, Braga, Portugal

Summary The taxonomy of the penicillia is unstable particularly in the important antibioticand mycotoxin-producing subgenus Penicillium. There are difficulties relating identifications to mycotoxin production. Also, the validity of dual nomenclature for pleomorphic fungi is under discussion increasingly. Patulin is an important mycotoxin produced by various fungi and has strict limits in the European Union. The mycotoxin and/or the isoepoxydon dehydrogenase (IDH) gene of the metabolic pathway have been assessed in 318 strains predominately of subgenus Penicillium. These data were used to classify the isolates. Subgenus Penicillium contained most of the IDH and patulin positives. The species and varieties in subgenus Penicillium which were associated with patulin detection can be reduced to one name, viz. Penicillium Pen $\mathrm{p}^{+}(\mathrm{p}=$ patulin). This has been extended to other mycotoxin producing penicillia to indicate the scope of the scheme. The classification will lead to the number of taxa being reduced, while avoiding species names and hence dual nomenclature. Culture independent analysis of environmental samples is mentioned. The scheme could be used with advantage for other fungi.

Key words Subgenus Penicillium, Patulin, Isoepoxydon dehydrogenase gene, Ochratoxin A, Mycotoxins, Identification, Environmental, Dual nomenclature

Species concepts within fungi are (a) subject to frequent revision and (b) unusually protean compared to other kingdoms [1]. Fungal classification, taxonomy and identifications are not sufficiently well studied for clear concepts of species to be revealed in many cases: They simply have not received the levels of attention given to other groups (i.e. plants, animals, and bacteria). Fungi have been demonstrated to be genetically closer to animals than plants, although they are recognised as a fifth kingdom. Also, the validity of dual nomenclature for pleomorphic fungi is under discussion increasingly in terms of whether to maintain the system that deviates from the one name for a species paradigm [6].

Names are desirable which are unambiguous in terms of exhibiting an important property in a biological sense (e.g. mycotoxin detection) [17] - they need to convey as much biological meaning as possible. A distinction is required between what (a) can be done conceivably (e.g. analysing virtually all secondary metabolites, and sequencing large numbers of genes) and (b) is useful and practical (e.g. analysing the property of most importance).

\author{
Corresponding address: \\ R. Russell M. Paterson \\ Micoteca da Universidade do Minho \\ Centro de Engenharia Biológica \\ Campus de Gualtar \\ 4710-057 Braga, Portugal \\ Tel.: + 351253604400 \\ Fax:+351253678 986 \\ E-mail: russell.paterson@deb.uminho.pt. \\ (2) 2006 Revista Iberoamericana de Micología \\ Apdo. 699, E-48080 Bilbao (Spain) \\ Apdo. 699, E-48080 B:
}

In addition, speciation issues have been ignored in the Fungi and have been described almost entirely in taxonomic terms based on descriptions of phenotypes - "morphospecies". However, many kind of biologically recognised groups such as races or special forms have been described of great practical significance. A high proportion of fungi are known only from the anamorphic state leading to preclusion from being recognised as species by some: Relationships to holomorphs are usually unclear. The information on speciation is still opaque [1]. Furthermore, the issue of cryptic species, or the very similar morphological "species" which are genetically different (e.g. Penicillium roqueforti, Penicillium carneum, and Penicillium paneum) needs to be addressed. Taxonomists usually adopt the view that very similar but not constantly different individuals be members of the same species: even if it is highly variable and polymorphic. This describes subgenus Penicillium well.

A satisfactory classification remains enigmatic for subgenus Penicillium which is particularly important because of antibiotic and mycotoxin production. Samson and Frisvad [18] have published recently a revision of the subgenus: It is reviewed in [9]. However, the systematics of the group has a history of cogitation (Paterson et al. [17]). The use of morphological characters which are only minutely and inconsistently different are the principal dilemmas. This has evolved into many taxonomic schemes and hence creating concomitant confusion. The names of fungi with important properties can be changed without adequate justification. Furthermore, existing taxonomic schemes were created before, or do not fully take into account, the requirements of the current mycotoxin era as described in CAST [2].

It needs to be recognised more generally by those not working directly in the field, that the primary reason that fungi are studied from food, feed and damp houses is 
for mycotoxin production (with all due weight given to organoleptic, allergic and opportunistic disease effects). However, it is almost perverse that it is within the mycotoxins area that so many problems arise of (a) variation in qualitative and quantitative production, and (b) numerous morphological variations within "species". It is because of these facts that the requirement for a simplified and yet more accurate, relevant and informative identification lie, especially in relation to detecting "weak spots" in commodity systems. The use of gene probes for metabolic pathways offers a protocol for establishing a firm taxonomy based on mycotoxin production potential. It is extremely useful if the mycotoxin of interest can also be detected to enable it to be determined if the gene is present and if it has been expressed. Also, a specific step in the biosynthetic pathway for an individual mycotoxin is ideal (e.g. isoepoxydon dehydrogenase) [14] compared to general steps such as polyketide synthestase, or even less satisfactory, marker genes unrelated to the particular mycotoxin biosynthetic pathway [21].

Paterson et al. [17] suggested a novel approach, and the concept was discussed further in $[9,14,16]$. Only identifications to a level of microscopy which can be attained readily are required combined with analysis for a particular mycotoxin. Ideally, this will include analysis for genes for metabolic pathways [21]. Ochratoxin A (OTA) is perhaps the most important mycotoxin of this taxon. It was suggested that, for example, instead of a Penicillium verrucosum (or Penicillium nordicum), "subgenus Penicillium OTAdetected" could be obtained. This can be contracted to Penicillium PenOTA ${ }^{+}$for example. In addition, the use of primers for the metabolic pathway may also be possible $[3,7,21]$.

Patulin is the second most important mycotoxin from the subgenus which had strict statutory limits imposed particularly in baby fruit-based foods by the European Union in 2004. Furthermore, it has a history of being studied as a model secondary metabolite and similarly can be a representative mycotoxin. However, the taxonomy of the producing fungi is particularly complicated $[14,18]$. Numerous penicillia have been analysed during the course of investigations on the isoepoxydon dehydrogenase (IDH) gene $[10,14,15]$. IDH is an important enzyme involved fundamentally in the metabolic pathway of patulin production [21]. Sufficient data have now accumulated to revise what constitutes patulin producing penicillia with a view to assisting quality control in food production. In addition, Paterson et al. [16] reported a scheme where patulin fungi could be identified as Penicillium Pen $\mathrm{p}^{+}$ and/or $\mathrm{IDH}^{+}$where $\mathrm{p}=$ patulin detection. Furthermore, direct identifications of "patulin fungi" in the environment are now a reality $[8,11]$ using culture independent PCR (CIP) [21]. The current paper (a) provides details of the scheme, (b) introduces more data, (c) and indicates how the procedures can be extended to other fungi.

\section{Material and methods}

The following provide details of how the patulin fungi were classified in relation to other fungi. Cultures, identifications and analyses were described in $[10,14,15]$, and are not repeated here. The primer sequences were always (IDH1): 5' CAA TGT GTC GTA CTG TGC CC; and (IDH2): 5'ACC TTC AGT CGC TGT TCC TC. However, some Penicillium brevicompactum and Penicillium bialowieziense strains from NRRL were analysed in a slightly modified manner. The IDH gene fragment was amplified by a variation of the protocol of [10]. The reac- tion buffer was that described by [20]. Primers were added at $0.5 \mu \mathrm{M}$ concentration and the thermal cycling profile was $96^{\circ} \mathrm{C}-2 \mathrm{~min}$, followed by 30 cycles of $96^{\circ} \mathrm{C}-30 \mathrm{sec}$, $52{ }^{\circ} \mathrm{C}-45 \mathrm{sec}, 72{ }^{\circ} \mathrm{C}-90 \mathrm{sec}$ and a final elongation reaction of $5 \mathrm{~min}$ at $72{ }^{\circ} \mathrm{C}$. Ten $\mu \mathrm{L}$ of each PCR reaction was loaded in a $1 \%$ agarose gel with TAE buffer, electrophoresed at a voltage gradient of 5 volts per centimetre for $60 \mathrm{~min}$, stained with ethidium bromide and visualize on a longwavelength UV trans-illuminator.

In addition, some Micoteca de Universidade do Minho (MUM), Portugal strains of Penicillium expansum (MUM 04.04, 04.10, 04.18, 04.28, 04.30, 04.32, 04.33, 04.36, 04.37, 04.06, 04.13) and P. brevicompactum (MUM $04.14,04.23,04.24,04.29,04.35)$ isolated from a bottled water factory were analysed. Federal Research Center for Nutrition, Germany strains of $P$. roqueforti (BFE 168, 169, $170,171,172,187,188,382,383$ ) were from blue cheese production and were analysed for patulin detection using one agar plug. Three plugs were used for the other strains. The PCR analysis was also slightly different: Strains were grown on malt extract agar (Peptone - Difco, USA - $1 \mathrm{~g}$; glucose - Merck, Germany - 20 g; malt extract, agar Oxoid, UK - $20 \mathrm{~g}$ each) and inoculated into $1 \mathrm{ml}$ glucose yeast malt [10] in Eppendorf tubes with continuous inversion for 3 days at room temperature (ca. $20^{\circ} \mathrm{C}$ ). The resulting pellet was removed by decanting. Reaction mixture: dNTPs $4 \mu \mathrm{l}(2.5 \mathrm{mM})$, buffer $5 \mu \mathrm{l}$, idh $14 \mu \mathrm{l}(25 \mathrm{mM})$, idh2 $4 \mu \mathrm{l}(25 \mathrm{mM})$, Taq DNA polymerase $0.25 \mu \mathrm{l}(50 \mathrm{U} / \mathrm{\mu l})$, $\mathrm{MgCl}_{2} 6 \mu \mathrm{l}(25 \mathrm{mM})$, Template DNA - $2.5 \mu \mathrm{l}$ (concentration varied depending on intensity of band by developing template DNA in an agar gel) $\mathrm{H}_{2} \mathrm{O}-50 \mu \mathrm{l}$. Cycling parameters (Mycycler - Bio-Rad, USA) were (a) $94{ }^{\circ} \mathrm{C}$ for $30 \mathrm{~min}$, (b) 30 cycles of $94^{\circ} \mathrm{C}$ for $1 \mathrm{~min}, 52^{\circ} \mathrm{C}$ for $1 \mathrm{~min}$, $72{ }^{\circ} \mathrm{C}$ for $2 \mathrm{~min}$ and (c) $72{ }^{\circ} \mathrm{C}$ for $5 \mathrm{~min}$. P. expansum MUM 04.04 was the positive control. A positive IDH result was a band slightly higher than 600 bp. A negative one was no band together with a positive band for the control. The isolated DNA was tested at dilutions of 0,15 and 150 to account for inhibition effects (see [14]) in the case of the BFE strains. Electrophoresis power pack was a Power-Pac 200 (Bio-Rad) set at 65-70 V. Temperature was ca. $20{ }^{\circ} \mathrm{C}$. The matrix was $1 \%$ (w/v) agarose $(0.8 \mathrm{Molecu}-$ lar Biology agarose, Bio-Rad). Strains which were positive or negative for the IDH gene were labeled $\mathrm{IDH}^{+}$or IDH $\mathrm{IDes}^{-}$ pectively, and similarly for patulin detection, i.e. pat $^{+}$and pat. In the case where strains had been analysed for both traits it was represented as IDH+ pat $^{+}$or $^{++}, \mathrm{IDH}^{-}$pat $^{-}$or $^{--}$, etc.

\section{Results}

The data for IDH and patulin detection are expressed in table 1. Many species possessed strains in more than one category (i.e. $\mathrm{IDH}^{+}, \mathrm{IDH}^{-}$, $\mathrm{pat}^{+}$, pat ${ }^{-}$, etc.). For example, $P$. roqueforti had representative in the positive and negative categories. Only $6 \%$ of $P$. brevicompactum were patulin positive and $3 \%$ of $P$. expansum were negative for both traits.

The IDH positive species were predominantly from subgenus Penicillium (64\%). Seventeen \% of Furcatum and Aspergilloides and none of the Biverticillium representatives were positive for the gene in comparison. The number of strains with and without the IDH gene in subgenus Penicillium were 127 and 71 respectively. Patulin detection was considerably lower than that for IDH. Penicillium was well represented in every grouping of IDH and patulin positive or negative (no strains fell in $\mathrm{IDH}^{-}$pat $^{+}$(i.e. ${ }^{+}$) although the lowest numbers were in the double negative category (Figure 1). There were small 
Table 1. IDH and patulin analyses of penicillia including the number of strains for each category in parenthesis, and the species included in each category.

\begin{tabular}{|c|c|c|}
\hline Subgenus & Category & Species \\
\hline \multirow[t]{7}{*}{ Penicillium } & $\mathrm{IDH}^{+} \mathrm{pat}^{+}(45)$ & P. brevicompactum, P. expansum, P. griseofulvum, P. griseofulvum var. dipodomyicola, P. roqueforti \\
\hline & $\mathrm{IDH}^{+}$pat $^{-}(43)$ & P. bialowieziense, P. brevicompactum, P. hirsutum, P. glandicola var. glandicola, P. glandicola var. glaucovenetum \\
\hline & $\mathrm{IDH}^{-}$pat- (31) & P. aurantiogriseum, $P$. chrysogenum, $P$. bialowieziense $P$. brevicompactum, $P$. expansum, $P$. griseofulvum, $P$. roqueforti \\
\hline & $\mathrm{IDH}^{+}(127)$ & $\begin{array}{l}\text { P. aurantiogriseum, P. bialowieziense, } P \text {. brevicompactum, } P \text {. expansum, } P \text {. griseofulvum, } P \text {. griseofulvum var. } \\
\text { dipodomyicola, P. hirsutum, P. glandicola var. glandicola, P. glandicola var. glaucovenetum, } P \text {. roqueforti }\end{array}$ \\
\hline & $\mathrm{IDH}^{-}(71)$ & $\begin{array}{l}\text { P. aurantiogriseum, } P \text {. chrysogenum, P. bialowieziense, P. brevicompactum, P. expansum, P. griseofulvum, P. hirsutum, } \\
\text { P. solitum, P. roqueforti }\end{array}$ \\
\hline & pat $^{+}(86)$ & P. brevicompactum, P. expansum, P. griseofulvum, P. griseofulvum var. dipodomyicola, P. roqueforti, \\
\hline & pat (91) & $\begin{array}{l}\text { P. aurantiogriseum, P. brevicompactum, P. bialowieziense, } P \text {. chrysogenum, } P \text {. expansum, } P \text {. freii, } P \text {. glandicola var. } \\
\text { glandicola, P. glandicola var. glaucovenetum, P. hirsutum, } P \text {. griseofulvum, P. roqueforti, P. solitum, P. viridactum }\end{array}$ \\
\hline \multirow[t]{7}{*}{ Furcatum } & $\mathrm{IDH}^{+} \mathrm{pat}^{+}(2)$ & P. paxilli \\
\hline & $\mathrm{IDH}^{+}$pat $^{-}(3)$ & P. paxilli, P. selandiae, P. simplicissimum \\
\hline & $\mathrm{IDH}^{-}$pat- (31) & P. citrinum, $P$. raistrickii, $P$. janczewskii, $P$. novae-zeelandiae, $P$. simplicissimum, $P$. waksmanii \\
\hline & $\mathrm{IDH}^{+}(10)$ & P. corylophilum, P. janczewskii, P. melinii, P. paxilli, P. selandiae, P. simplicissimum \\
\hline & $\mathrm{IDH}^{-}(50)$ & P. canescens, P. citrinum, P. raistrickii, P. janczewskii, P. novae-zeelandiae, P. paxilli, P. simplicissimum, P. waksmanii \\
\hline & $\operatorname{pat}^{+}(3)$ & P. janczewskii, P. paxilli \\
\hline & pat (42) & $\begin{array}{l}\text { P. citrinum, P. corylophilum, P. janczewskii, P. novae-zeelandiae, P. paxilli, P. raistrickii, P. selandiae, P. simplicissimum, } \\
\text { P. waksmanii }\end{array}$ \\
\hline \multirow[t]{4}{*}{ Aspergilloides } & $\mathrm{IDH}^{-}$pat $^{-}(5)$ & P. glabrum, P. restrictum \\
\hline & $\mathrm{IDH}^{+}(1)$ & P. spinulosum \\
\hline & $\mathrm{IDH}^{-}(5)$ & P. glabrum, $P$. restrictum \\
\hline & pat (10) & P. glabrum, P. restrictum, P. spinulosum \\
\hline \multirow[t]{3}{*}{ Biverticillium } & $\mathrm{IDH}^{-}$pat $^{-}(3)$ & P. purpurogenum \\
\hline & $\mathrm{IDH}^{-}(1)$ & P. pinophilum, \\
\hline & pat (4) & P. purpurogenum, P. rubrum \\
\hline None & $\mathrm{IDH}^{-}$pat $^{+}$ & \\
\hline
\end{tabular}

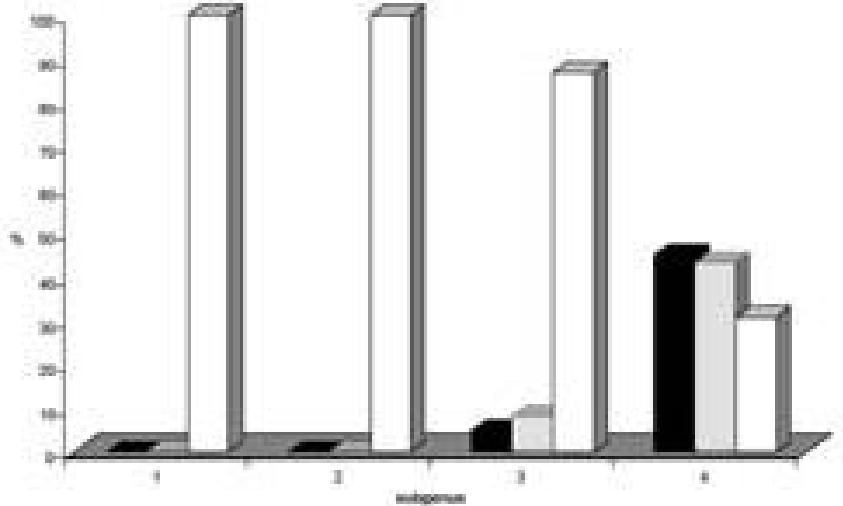

Figure 1. Percentage of strains tested for the IDH gene and patulin in Aspergilloides (1), Biverticillium (2), Furcatum (3), and Penicillium (4). Black

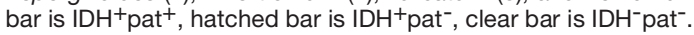

percentages of ${ }^{++}$and ${ }^{+-}$strains in Furcatum. The other subgenera were represented as ${ }^{--}$. The species which were included in the various categories had diverse names and were frequently included in different groups. For example, $P$. brevicompactum was in ${ }^{++},{ }^{+-},{ }^{--}$. There were no P. expansum ${ }^{+-}$.

\section{Discussion}

The presence and absence of the IDH gene and patulin detection within the same (morpho) species is of interest. It indicates that the conventional method is unreliable for a number of potential reasons such as the existence of cryptic species, misidentifications, variation in characters, and contamination. The proposed identification is that strains in subgenus Penicillium are referred to by giving the genus name, then the subgenus name followed
Table 2. The identifications based on the analysis of the IDH gene and patulin.

\begin{tabular}{|c|c|}
\hline Subgenus & The identification \\
\hline \multicolumn{2}{|l|}{ Penicillium } \\
\hline \multicolumn{2}{|l|}{ IDH only } \\
\hline $\mathrm{IDH}^{+}$ & Penicillium PenIDH ${ }^{+}$ \\
\hline $\mathrm{IDH}^{-}$ & Penicillium PenIDH' \\
\hline \multicolumn{2}{|c|}{ patulin only } \\
\hline pat $^{+}$ & Penicillium Penpat ${ }^{+}$ \\
\hline pat $^{-}$ & Penicillium Penpat ${ }^{-}$ \\
\hline \multicolumn{2}{|c|}{ IDH and patulin } \\
\hline $\mathrm{IDH}^{+}$pat $^{+}$ & Penicillium PenIDH ${ }^{+}$pat $^{+}$ \\
\hline $\mathrm{IDH}^{+}$pat $^{-}$ & Penicillium PenIDH ${ }^{+}$pat $^{-}$ \\
\hline $\mathrm{IDH}^{-}$pat $^{-}$ & Penicillium PenIDH'pat ${ }^{-}$ \\
\hline \multicolumn{2}{|l|}{ Furcatum ${ }^{\star}$} \\
\hline \multicolumn{2}{|l|}{ IDH only } \\
\hline $\mathrm{IDH}^{+}$ & Penicillium FurIDH ${ }^{+}$ \\
\hline $\mathrm{IDH}^{-}$ & Penicillium FurIDH ${ }^{-}$ \\
\hline \multicolumn{2}{|c|}{ patulin only } \\
\hline pat $^{+}$ & Penicillium Furpat $^{+}$ \\
\hline pat $^{-}$ & Penicillium Furpat $^{-}$ \\
\hline \multicolumn{2}{|c|}{ IDH and patulin } \\
\hline $\mathrm{IDH}^{+}$pat $^{+}$ & Penicillium FurIDH ${ }^{+}$pat $^{+}$ \\
\hline $\mathrm{IDH}^{+}$pat $^{-}$ & Penicillium FurIDH ${ }^{+}$pat $^{-}$ \\
\hline $\mathrm{IDH}^{-}$pat $^{-}$ & Penicillium FurIDH-pat ${ }^{-}$ \\
\hline
\end{tabular}

*in practice it is suggested that Furcatum is grouped as "biverticillate" with Biverticillium Other Subgenera can be substituted as appropriate.

by the property tested and whether it was positive or negative (Table 2). This is intended to be of particular use to the food industry rather than as a novel taxonomy, although a platform is provided inevitably for further discussion in that area. For example, Penicillium Penicillium $\mathrm{IDH}^{+}$is abbreviated to Penicillium $\mathrm{PenIDH}^{+}$in the case of the IDH gene. Similarly those which are patulin positive 
Table 3. Proposed identification scheme based on mycotoxin detection extended to other mycotoxins in the subgenus Penicillium.

\begin{tabular}{|c|c|c|c|}
\hline Mycotoxin & $\begin{array}{l}\text { Importance as } \\
\text { mycotoxin* }\end{array}$ & Producing species (Frisvad et al. 2004) & $\begin{array}{l}\text { Proposed } \\
\text { identification }\end{array}$ \\
\hline asteltoxin & C & P. cavernicola, P. concentricum, P. confertum, P. formosanum, P. tricolor & Penicillium Pen $\mathrm{a}^{+}$ \\
\hline chaetoglobosin A & $\mathrm{B}$ & P. discolar, $P$. expansum, $P$. marinum & Penicillium Pen ca ${ }^{+}$ \\
\hline citrinin & $B$ & P. expansum, P. radicicola, $P$. verrucosum & Penicillium Pen $\mathrm{c}^{+}$ \\
\hline -cyclopiazonic acid & B & P. palitans, P. griseofulvum, P. dipodomyicola, P. commune, $P$. camemberti & Penicillium Pen rr $^{+}$ \\
\hline griseofulvin & C & $\begin{array}{l}\text { P. aethiopicum, P.corprophilum, P. dipodomyicola, P. griseofulvum, P. persicinum, } \\
\text { P. sclerotigenum }\end{array}$ & Penicillium Pen $\mathrm{g}^{+}$ \\
\hline mycophenolic acid & B & P. bialowieziense & Penicillium Pen $\mathrm{m}^{+}$ \\
\hline nephrotoxic glycopeptides & $\mathrm{B}$ & P. aurantiogriseum, P. polonicum & Penicillium Pen $\mathrm{n}^{+}$ \\
\hline ochratoxin A & A & P. nordicum, P. verrucosum & Penicillium Pen $\mathrm{O}^{+}$ \\
\hline patulin & A & $\begin{array}{l}\text { P. carneum, P. clavigerum, P. concentricum, P. coprobium, P. dipodomyicola, } \\
\text { P. expansum, P. gladioli, P. griseofulvum, P. marinum, P. paneum, P. sclerotigenum, } \\
\text { P. vulpinum }\end{array}$ & Penicillium Pen $\mathrm{p}^{+}$ \\
\hline penicillic acid & B & $\begin{array}{l}\text { P. aurantiogriseum, P. carneum, } P . \text { cyclopium, } P \text {. freii, } P . \text { melanoconidium, } \\
\text { P. neoechinulatum, P. polonicum, P. radicicola, P. tulipae, P. viridicatum }\end{array}$ & Penicillium Pen pa ${ }^{+}$ \\
\hline penicillins & $\mathrm{C}$ & P. chrysogenum, P. dipodomyis, P. flavigenum, P. griseofulvum, P. nalgiovense & Penicillium Pen pe ${ }^{+}$ \\
\hline PR toxin & $\mathrm{B}$ & P. chrysogenum, P. roqueforti & Penicillium Pen $\mathrm{pr}^{+}$ \\
\hline penitrem A & B & $\begin{array}{l}\text { P. carneum, P. clavigerum, P. crustosum, P. flavigenum, P. glandicola, P. granulatum, } \\
\text { P. melanoconidium, P. tulipae }\end{array}$ & Penicillium Pen $\mathrm{pt}^{+}$ \\
\hline roquefortine C & C & 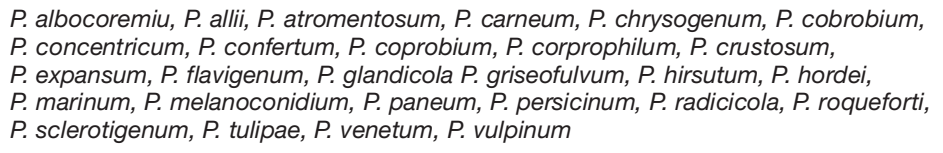 & Penicillium Pen $\mathrm{r}^{+}$ \\
\hline territrems & C & P. cavernicola, P. echinulatum & Penicillium Pen $\mathrm{t}^{+}$ \\
\hline viridicatum toxin & B & P. aethiopicum & Penicillium Pen vt ${ }^{+}$ \\
\hline viriditoxin & C & P. mononematosum & Penicillium Pen v+ \\
\hline xanthomegnin and others & $\mathrm{B}$ & $\begin{array}{l}\text { P. clavigerum, P. cyclopium, P. flavigenum, P. freii, P. melanoconidium, P. tricolour, } \\
\text { P. viridicatum }\end{array}$ & Penicillium Pen $\mathrm{x}^{+}$ \\
\hline
\end{tabular}

${ }^{*} \mathrm{~A}=$ high, $\mathrm{B}=$ moderate, $\mathrm{C}=$ some (approximate classification)

will be Penicillium Penp ${ }^{+}$(where " $\mathrm{p}$ " is the abbreviation of patulin). A Latin binomial is not implied by this identification with relevance to giving single names to pleomorphic fungi: Obviously an isolate could be identified conventionally to establish the holomorph, e.g. by nucleic acid analysis.

The classification of the $P$. roqueforti strains are of interest (Table 1). There were two groups: (a) IDH and patulin positive and (b) IDH and patulin not detected. The positives were from orchards and the negatives from cheese. The orchards strains may conform to $P$. paneum and/or $P$. carneum as described in [18] because of the ability to produce detectable patulin. It is unlikely that the $P$. roqueforti strains from orchards could have been recognised as the other two species on the basis of the methods employed. The cheese strains may be $P$. roqueforti, although the report of patulin-producing $P$. roqueforti from blue cheese requires consideration [5]. On the other hand, patulin was not considered at all in [19] where $P$. roqueforti and $P$. paneum were identified from silage indicating considerable confusion.

It is emphasised that is not simply the morphological identifications that can be rationalised. The analysis for the metabolite of interest (e.g. patulin) can also be, so that specific techniques can be used for the compound of interest rather than general ones (e.g. those in [2] cf. [18] respectively). In effect, a more objective identification is obtained which relates to a particular problem (e.g. the determination of weak spots within a commodity production system [17]). The development of genetic analysis of metabolic pathways involved in the production of particular mycotoxins has increased horizons in mycotoxicology including taxonomy [21]. It is possible to determine whether mycotoxin production within a fungus is possible or not. Chromatographic methods can only reveal if a compound is detected and so are dependant on detection limits. However, potential limitations of the PCR must also be realised such as inherent detection limits, contamination [13], inhibition, and DNA alterations [14]; it is not a catholicon [21].

There are approximately 18 other mycotoxins (as named in [18]) for which a similar treatment can be devised (Table 3). Obviously, a standardised form of abbreviation would be required for a usable name if many compounds were detected from a single strain (e.g. patulin to "p", citrinin to "c", etc.). A similar approach could be employed for gene probes with the abbreviation being underlined or italicised for example. In this way the most toxic penicillia will be identified as defined by [18]

In three cases only is one toxin associated with a single species (i.e. P. bialowiezense, Penicillium aethiopicum, and Penicillium mononematosum) whereas, at the other extreme, numerous species are (e.g. those associated with roquefortine $\mathrm{C}$ ). In the latter case an extremely large number of species (representing a large amount of work to identify) are reduced to one "name". There are similar examples of other mycotoxins which have large numbers of names associated with their production. Most of the mycotoxins can be detected by uncomplicated chromatographic techniques, although more sophisticated methods could be employed. Furthermore, if the objective is to find weak spots in a commodity system (e.g. a particular food) then separation into the various subgenera may be unnecessary. It is the user of the scheme that will decide. Of considerable appeal are the PCR based methods [21] and table 4 indicates where there may be sufficient genetic information for primers to be devised [4,7].

As mentioned, it is likely that some isolates will produce detectable amounts of more than one of the mycotoxins listed here. Obviously, the names of the multiple mycotoxin producers can simplified to include the additional compounds e.g. P. pen cit $^{+}$ota $^{+}$, etc which be abbrevia- 
Table 4. Proposed identification based on possession of a gene for mycotoxin production where there may be sufficient information in the literature to devise primers for analysis.

\begin{tabular}{ll}
\hline Mycotoxin & Gene based identification \\
\hline Ochratoxin A & Penicillium Pen ota ${ }^{+}$ \\
Patulin & Penicillium Pen idh $^{+}$ \\
Penicillins & Penicillium Pen pen ${ }^{+}$ \\
PR toxin & Penicillium Pen prt ${ }^{+}$ \\
\hline
\end{tabular}

ted even further e.g. cit to "c" and ota to "o", to avoid excessively large names: A large number of mycotoxins are detectable from some individual species e.g. five from $P$. griseofulvum. References to general analytic screening methods for these mycotoxins are provided in [12], although specific methods are optimal for enhancing detection limits.

By using the 18 mycotoxins listed in table 3, 90\% of the 58 species in [18] can be identified as they are reported to produce detectable amounts of at least one of these compounds. Fifty four percent of the $90 \%$ had a unique pattern. However, some species share one or more of the mycotoxins: There were (a) four cases of two species, (b) three cases of three species, and (c) one case of seven species sharing compounds. For examples, roquefortine $\mathrm{C}$ and patulin were in common for Penicillium coprobium, $P$. paneum, and Penicillium vulpinum: These are reduced to Penicillium pen $\mathrm{r}^{+} \mathrm{p}^{+}$. Penicillium albocoremium, Penicillium allii, Penicillium atromentosum, Penicillium cobrobium, Penicillium hirsutum, Penicillium hordei, and Penicillium venetum produce roquefortine $\mathrm{C}$ and are $P$. pen $\mathrm{r}^{+}$following the same logic.
In summary, when a culture is received for identification the following can be undertaken:

1. Determine if the fungus is a Penicillium by examining the conidiophore,

2. Determine if the Penicillium is terverticillate

3. Undertake analysis for the mycotoxin and/or the particular mycotoxin gene

Some species of other genera will also produce the particular mycotoxin (e.g. Aspergillus ochraceus and OTA): It is the responsibility of the particular workers how to approach these other possibilities. The scheme could be applied to other fungi.

This form of identification can be extended to environmental or "virgin" samples by using CIP [21]. Paterson et al. [8] demonstrated that the gene fragment could be identified from such orchard samples and Paterson [11] indicated, inter alia, that non orchard soil did not possess the gene whereas some orchard ones did.

In conclusion, an identification of the penicillia is afforded which (a) is consummated readily, (b) is objective, (c) contributes to "taxa deflation", and (d) provides information relevant directly to the present mycotoxin era.
Russell Paterson is grateful for grant

SFRH/BPD/14923/2004 from Fundação para a

Ciência e a Tecnologia, Portugal.

\section{References}

1. Burnett J. Fungal Populations and Species. Oxford, Oxford University Press, 2003.

2. CAST. Mycotoxins: Risks in plant, animal, and human systems. Ames, Council for Agricultural Science and Technology, 2003. Agricultural Science

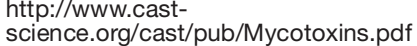
science.org/

3. Dao HP, Mathieu F, Lebrinhi A. Two primers to detect OTA producers by PCR method. Int J Food Microbiol 2005; 104: 61-67.

4. Edwards SG, O'Callaghan J, Dobson ADW. PCR- based detection and quantification of mycotoxigenic fungi. Mycol Res 2002; 106: 1005-1025.

5. Erdogan A, Sert S. Mycotoxin-forming ability of two Penicillium roqueforti strains in blue moldy Tulum cheese ripened at various temperatures. J Food Protect 2004; 67: 533-535.

6. Hawksworth DL. Limitation of dual nomenclature for pleomorphic fungi. Taxon 2004; 53: 596-598.

7. Karolewiez A, Geisen R. Cloning a part of the ochratoxin $A$ biosynthetic gene cluster of Penicillium nordicum and characterising of the ochratoxin polyketide gene. Syst Appl Microbiol 2005; 28: 588-595.

8. Paterson RRM, Archer S, Kozakiewicz Z, Lea A, Locke T, O'Grady, E. A gene probe for the patulin metabolic pathway with potential use in novel disease control. Biocontrol Sci Technol 2000; 10: 509-512.

9. Paterson RRM. Book Review. Mycotaxon 2005; 91: 519-521.
10. Paterson RRM, Kozakiewicz Z, Locke T, Brayford D, Jones SCB. Novel use of the isoepoxydon dehydrogenase gene probe of the patulin metabolic pathway and chromatography to test penicillia isolated from apple production systems for the potential to contaminate apple juice with patulin. Food Microbiol 2003; 20: 359-364.

11. Paterson RRM. Primers from the isoepoxydon dehydrogenase gene of the patulin biosynthetic pathway to indicate critical control points for patulin contamination of apples. Food Control 2006; 17: 741-744.

12. Paterson RRM. Standardized data on 474 metabolites or "extrolites". Mycol Res 2004; 108: 596-597.

13. Paterson RRM. The coronamycin producer: a case of mistaken identity? Mycol Res 2005; 109: 850-851.

14. Paterson RRM. The isoepoxydon dehydrogenase gene of patulin biosynthesis in cultures and secondary metabolites as candidate PCR inhibitors. Mycol Res 2004; 108: 1431-1437.

15. Paterson RRM. The isoepoxydon dehydrogenase gene of the patulin biosynthetic pathway, patulin detection, and the utility of species names in patulin-producing fungi. In: Lima N, Smith D (Eds.) Biological resource centres and the use of microbes. Braga Micoteca da Universidade do Minho, 2003: 259-266.
16. Paterson RRM, Venâncio A, Lima N. An applied identification scheme from Penicillium strains by patulin and isoepoxydon dehydrogenase gene detection with utility in HACCP control using "virgin" samples. Microbes in a Changing World, XI International Congress of Mycology, San Francisco, 2005; 9.

17. Paterson RRM, Venâncio A, Lima N. Solutions to Penicillium taxonomy crucial to mycotoxin research and health. Res Microbiol 2004; 155: 507-513.

18. Samson RA, Frisvad JC. Penicillium subgenus Penicillium: New taxonomic subgenus Penicillium: New taxonomic Stud Mycol 2004; 49: 1-258.

19. Sumarah MW, Miller JD, Blackwell BA. Isolation and metabolite production by Penicillium roqueforti, $P$. paneum and $P$. crustosum isolted in Canada. Mycopathologia 2005; 159: 571-577.

20. White TJ, Bruns TD, Lee SB, Taylor JW. Amplification and direct sequencing of fungal ribosomal DNA for phylogenetics. In: Innes MA, Gelfand DH, Sninsky JJ, White TJ (Eds.) PCR Protocols: A guide to the methods and applications. New York, Academic Press, 1990: 315-322.

21. Paterson RRM. Identification and quantification of mycotoxigenic fungi by PCR. Proc Biochem 2006; 41: 1467-1474. 